\title{
New insight into vascular homeostasis and injury-reconstruction
}

\author{
ZHANG YouYi ${ }^{1 *} \&$ DONG ErDan ${ }^{2 *}$ \\ ${ }^{1}$ Institute of Vascular Medicine, Peking University Third Hospital, Key Laboratory of Cardiovascular Molecular Biology and Regulatory \\ Peptides and Beijing Key Laboratory of Cardiovascular Receptors Research, Beijing 100191, China; \\ ${ }^{2}$ Department of Health Sciences, National Natural Science Foundation of China, Beijing 100085, China
}

Received July 18, 2014

Citation: Zhang YY, Dong ED. New insight into vascular homeostasis and injury-reconstruction. Sci China Life Sci, 2014, 57: 739-741, doi: $10.1007 / \mathrm{s} 11427-014-4719-\mathrm{x}$

Acquired vascular abnormalities are a pivotal part of the pathophysiology of multiple cardiovascular and metabolic diseases. Complications of various types of vascular pathology, for example, stroke, myocardial infarction and aortic dissection constitute most common causes of disability and deaths worldwide [1]. Ageing per se is also associated with significant remodeling of vasculatures that exacerbates concomitant cardiovascular or metabolic diseases [2]. Important discoveries in the field of vascular biology have enhanced our understanding of functional and morphological abnormalities of vasculatures. It is both critical and timely to establish preventive and curative measures to mitigate major vascular abnormalities. Blood vessels are tube-shape organs made of endothelium, smooth muscle and fibroblast cells. Large vessels function as conduit channel for blood transport while small vasculatures form networks to delivery nutrients to all other organs in the body. Blood vessels also possess the function of sensing changes of internal environment, forming a barrier to particular organ or tissues such as brain, integrating and transmitting signals and producing bioactive molecules to regulate vascular homeostasis, regeneration and remodeling.

Major cardiovascular and cerebrovascular diseases frequently form frontier scientific issues for that the regulation of vascular homeostasis and injury-reconstruction. The auto-homeostasis of the functionality of blood vessels is an important basis of body activities, and plays a key role in ensuring physiological activities of the body. Restructuring

*Corresponding author (email: zhangyy@bjmu.edu.cn; donged@mail.nsfc.gov.cn) of blood vessels is an active process changes in vascular structure, and consists of the growth, death and migration of vascular cells [3], as well as the generation and degradation of extracellular matrix [4]. Vascular remodeling is an adaptive physiological process which maintains the homeostasis of blood vessels, and also a key and common pathological entity for many cardiovascular and metabolic diseases.

Sixteen groups of researchers have contributed articles to this spotlight issue. Novel discoveries and cutting-edge knowledge in molecular biology, pathophysiology, biomechanics, genomics, epigenetics, bioinformatics of vascular homeostasis and vascular diseases are highlighted in this thematic issue.

Calorie restriction is one of the new topics in cardiovascular research. Calorie restriction is a dietary regime based on low calorie intake. The role of calorie restriction in humans is controversial. Recently, new data have provided new insights into the potential role of calorie restriction in vascular homeostasis, including reduced oxidative stress, enhanced nitric oxide bioactivity, and decreased inflammation. Liu DePei's group reviewed the role of some important molecules, including sirtuins, AMP-activated protein kinase, mammalian targets of rapamycin, endothelial nitric oxidase and their regulatory pathways on the maintenance of vascular homeostasis. Endothelial mechanosensors, as gatekeepers of vascular homeostasis and adaptation under mechanical stress, has come into notice recent years. Endothelial cells serve as a barrier between blood and extravascular space, but also a sensor for mechanical stimulus as well as 
contributor to the pathogenesis of vascular disorders. Luo JinCai et al. focused on recent findings in endothelial mechanosensors in subcellular microdomains and their roles in regulation of physiological and pathological functions under mechanical stress in their review.

The development and differentiation of endothelial cells is a critical physiological process for vascular homeostasis. Differentiation of stem cells into endothelial cells is a popular topic again. We can get more current understanding of how stem cells differentiate into endothelial cells at the level of signaling, transcription and microRNA regulation. The authors also summarized recent advances of Notch pathway with special emphasis on the underlying molecular mechanisms and the effects of intermedin (adrenomedullin 2), a peptide related to the calcitonin gene-related peptide (CGRP) family, on the maintaining vascular homeostasis.

As a common knowledge, inflammation is one of most important pathological response which is involved in vascular injury and repair, especially adverse remodeling. Some new concepts are demonstrated by accumulating research articles. Complement component 5a is an important proinflammatory mediator on activation of the complement cascade. Recently, more evidence shows that the complement component $5 \mathrm{a}$ is a significant pathogenic driver of arteriosclerotic vascular lesions. Also immune cells, T lymphocytes are involved in formation of aortic aneurysms. The renin-angiotensin system (RAS) regulates vascular tone and plays a critical role in vascular remodeling. The authors focus on angiotensin converting enzyme 2, a negative regulator of the RAS. Increasing evidences indicate that ACE2 may be a novel therapy target for vascular disorder and remodeling. Mineralocorticoid receptor (MR) is a classic nuclear receptor. MR mediates inflammatory reaction and oxidative stress and therefore exacerbates vascular remodeling. MR antagonists can inhibit these effects. More studies will bring to light the molecular mechanisms to support this notion. Intracellular $\mathrm{Ca}^{2+}$ homeostasis is essential for vascular function and blood pressure regulation. A novel class of non-selective cation channels, called transient receptor potential (TRP) channels, have emerged at the frontier of hypertension research. Liu DaoYan et al. summarized the functions of two types of TRP channels and proposed that the imbalance of TRP channels function may be a new etiology for the pathogenesis of hypertension.

At present, emerging evidence strongly suggests that epigenetic modifications may play a role in the developmental blood vessels and maintain vascular homeostasis and are also involved in vascular remodeling. Several types of non-coding RNA, such as microRNAs (miRNAs) and long non-coding RNAs (lncRNAs), are involved in regulating the endothelial cell and vascular smooth muscle cell (VSMC) behaviors. In this issue, Jing Qing et al. introduced the in- teraction between miRNAs and IncRNAs, and highlighted the mechanism through which lncRNAs regulate the miRNA function. Xin HongBo et al. summarized the latest insights into the roles of RNA-binding proteins in controlling vascular endothelial cell functions and their contribution to the pathogenesis of vascular inflammatory diseases. Wu YongJian and his colleagues presented an overview on the discovery of "metabolic memory", the recent progress in its molecular mechanisms, and epigenetic modifications. Cui QingHua's group presented a genomic location based bioinformatics method to predict the lncRNAs associated with vascular disease. The result suggests that the presented method has a reliable prediction performance.

Ageing is the accumulation of changes in a person over time. Senescence refers to cellular senescence or senescence of the whole organism. It is commonly believed that cellular senescence is causally implicated in generating age-related phenotypes, and removal of senescent cells can prevent or delay tissue dysfunction and extend health span [5]. In the last two reviews, Zhang WeiLi et al. introduced the potential roles of leukocyte telomere length dynamics in the imbalance between injurious oxidative stress/inflammation and endothelial repair during the pathogenesis of agerelated atherosclerosis, and Wang WenGong discussed the current knowledge of HuR's role in vascular cell senescence and vascular aging with their studies.

These reviews bring us brand-new information and conception for vascular homeostasis and injury-reconstruction. It is helpful for understanding the mechanism of vascular homeostasis regulation and injury-reconstruction and therefore for looking for early diagnosis of disease and disease outcomes warning signs.

This work was supported by the National Natural Science Foundation of China (91339000).

1 World Health Organization. The 10 leading causes of death in the world, 2000 and 2012. 2014, available from http://www.who.int/ mediacentre/factsheets/fs310/en/

2 Bachschmid MM, Schildknecht S, Matsui R, Zee R, Haeussler D, Cohen RA, Pimental D, Loo BV. Vascular aging: chronic oxidative stress and impairment of redox signaling-consequences for vascular homeostasis and disease. Ann Med, 2013, 45: 17-36

3 Yu H, Clarke MC, Figg N, Littlewood TD, Bennett MR. Smooth muscle cell apoptosis promotes vessel remodeling and repair via activation of cell migration, proliferation, and collagen synthesis. Arterioscler Thromb Vasc Biol, 2011, 31: 2402-2409

4 Chen Q, Jin M, Yang F, Zhu J, Xiao Q, Zhang L. Matrix metalloproteinases: inflammatory regulators of cell behaviors in vascular formation and remodeling. Mediators Inflamm, 2013, 2013: 928315

5 Baker D, Wijshake T, Tchkonia T, LeBrasseur N, Childs B, van de Sluis B, Kirkland J, van Deursen J. Clearance of p16Ink4a-positive senescent cells delays ageing-associated disorders. Nature, 2011, 479: 232-236

Open Access This article is distributed under the terms of the Creative Commons Attribution License which permits any use, distribution, and reproduction in any medium, provided the original author(s) and source are credited. 


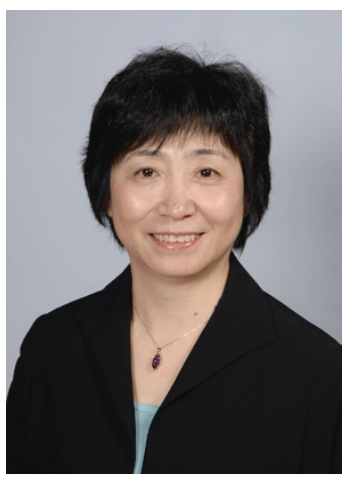

\section{Biographical Sketch}

Zhang YouYi, Professor in Medicine, Director of Institute of Cardiovascular Science, Peking University; Associate Director of Institute of Vascular Medicine, Peking University Third Hospital; vice Dean of Academy for Advanced Interdisciplinary Studies Peking University. Dr. Zhang obtained her B.S. in Medicine in 1982 and Ph.D. at Xinjiang Medical University and Nihon University in 1992. She worked in Institute of Vascular Medicine at Peking University Third Hospital as a post-doctoral fellow between 1992 and 1994. She worked as a post-doctoral fellow at the Department of Pharmacology, Emory University, USA from 1999 to 2001. Since 1993, her research has been focused on adrenoceptors and cardiovascular disease, especially on distribution, expression, mediated biological function, signal transduction, regulation, cross-talk between adrenoceptors, and pathophysiological alterations of adrenoceptors in heart and blood vessels. Recently she started to work on study of the signaling of the cardiac adrenoceptors in heart remodeling and heart failure as well as single molecular imaging detection in living cells. As a principle investigator, she was in charge of more than ten grants including the National Basic Research Program of China (973 Program) from Ministry of Science and Technology, and grants from the National Natural Science Foundation of China. She has published 200 research articles, 115 of which were in international journals.

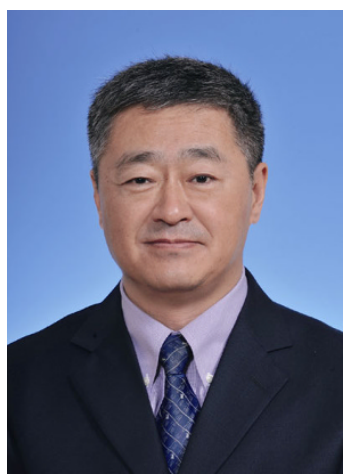

\section{Biographical Sketch}

Dong Erdan, MD, Ph.D., Professor of Medicine, Executive Director of Department of Health Sciences of National Natural Science Foundation of China. Dr. Dong graduated from Peking University, and conducted his post-doctoral research in University of Rochester, USA. He has long been engaged in basic cardiovascular research and scientific fund management. He has hosted or participated in many programs of the National Natural Science Foundation of China and National Basic Program of China (973 Program), and published about 100 papers. He is Vice Editor-in-Chief of Chinese Journal of Hypertension, and member of editorial boards of more than 10 journals, including The Chinese Medical Journal, Journal of Geriatric Cardiology, Science Foundation in China, and Science China Life Sciences. He is member of the evaluation committee of National Award for Progress in Science and Technology and State Natural Science Award, and member of Special Committee for Academic and Society affairs of China Association for Science and Technology. He has won awards five times and honors twice from the National Natural Science Foundation of China, and enjoyed special government allowances from the State Council in 2012. 